\title{
Dimension and codimension of simple games
}

\author{
Sascha Kurz \\ Department of Mathematics \\ University of Bayreuth \\ Germany \\ sascha.kurz@uni-bayreuth.de \\ Xavier Molinero* \\ Department of Mathematics \\ Universitat Politècnica de Catalunya \\ Spain \\ xavier.molinero@upc.edu \\ Martin Olsen \\ Department of Business Development and Technology \\ BTECH, Aarhus University \\ Denmark \\ martino@btech.au.dk \\ Maria Serna ${ }^{\dagger}$ \\ Department of Computer Science \\ Universitat Politècnica de Catalunya \\ Spain \\ mjserna@cs.upc.edu
}

\begin{abstract}
This paper studies the complexity of computing a representation of a simple game as the intersection (union) of weighted majority games, as well as, the dimension or the codimension. We also present some examples with linear dimension and exponential codimension with respect to the number of players.
\end{abstract}

Keywords: Simple games, Dimension, Codimension, Computational complexity

MSC: 91B12, 91A12

\footnotetext{
*Partially funded by grant MTM2012-34426/FEDER from MINECO and FEDER.

${ }^{\dagger}$ Partially funded by grant TIN2013-46181-C2-1-R from MINECO and FEDER and by grant 2014SGR1034 (ALBCOM) of "Generalitat de Catalunya".
} 


\section{Introduction and preliminaries}

We consider the so-called simple games and the computational complexity of representing them as unions or intersections of weighted majority games. Simple games and its dimension, as well as, weighted majority games, were defined by Taylor and Zwicker [12]. Later, Freixas and Marciniak [3] introduced a new concept, the codimension of simple games.

A simple game is a tuple $\Gamma=(N, \mathcal{W})$, where $N$ is a finite set of players and $\mathcal{W} \subseteq \mathcal{P}(N)$ is a monotonic family of subsets of $N$. Furthermore, its dual $\Gamma^{*}=\left(N, \mathcal{W}^{*}\right)$ is the game such that $\mathcal{W}^{*}=\{S \subseteq N: N \backslash S \notin \mathcal{W}\}$. $\Gamma$ is said to be self-dual if $\Gamma=\Gamma^{*}$. Note that $\left(\Gamma^{*}\right)^{*}=\Gamma$. Given two simple games $\Gamma_{1}=\left(N_{1}, \mathcal{W}_{1}\right)$ and $\Gamma_{2}=\left(N_{2}, \mathcal{W}_{2}\right)$, they are equivalent if $N_{1}=N_{2}$ and $\mathcal{W}_{1}=\mathcal{W}_{2}$. The subsets of $N$ are called coalitions, the set $N$ is the grand coalition and each $X \in \mathcal{W}$ is a winning coalition. The complement of the family of winning coalitions is the family of losing coalitions $\mathcal{L}$, i.e., $\mathcal{L}=\mathcal{P}(N) \backslash \mathcal{W}$. Any of those set families determine uniquely the game $\Gamma$ and constitute one of the usual forms of representation for simple games [12], although the size of the representation is not, in general, polynomial in the number of players [10].

A simple game $\Gamma$ is a weighted majority game (WMG) if it admits a representation by means of $n+1$ nonnegative real numbers $\left[q ; w_{1}, \ldots, w_{n}\right]$ such that $S \in \mathcal{W} \Longleftrightarrow w(S) \geq q$ where, for each coalition $S \subseteq N, w(S)=$ $\sum_{i \in S} w_{i}$. The number $q$ is called the quota and $w_{i}$ the weight of the player $i$. It is well known that any WMG admits a representation with integer numbers.

The dimension of a simple game $\Gamma$ is the least $k$ such that there exists WMGs $\Gamma_{1}, \ldots, \Gamma_{k}$ such that $\Gamma=\Gamma_{1} \cap \ldots \cap \Gamma_{k}$. On the other hand, the codimension of a simple game $\Gamma$ is the least $k$ such that there exists WMGs $\Gamma_{1}, \ldots, \Gamma_{k}$ such that $\Gamma=\Gamma_{1} \cup \ldots \cup \Gamma_{k}$.

Many theoretical results and examples about dimension and codimension $[11,8,3,7,5,12,6]$ have been constantly appearing during the last years, as well as, computational complexity results $[9,4,1]$. We present some results that will be used in Section 2.

Lemma 1. The dimension of a simple game $v$ is bounded above by $\left|\mathcal{L}^{M}\right|$ and the co-dimension is bounded above by $\left|\mathcal{W}^{m}\right|$.

Lemma 2. Let $\Gamma$ be a simple game. $\Gamma$ is the intersection of $t$ weighted games if and only if $\Gamma^{*}$ is the union of $t$ weighted games. Furthermore a representation, as union (intersection), of $\Gamma^{*}$ can be obtained from a representation, as intersection (union), of $\Gamma$ in polynomial time. Moreover, $\operatorname{dim}(\Gamma)=\operatorname{codim}\left(\Gamma^{*}\right)$, and if $\Gamma$ is self-dual then $\operatorname{dim}(\Gamma)=\operatorname{codim}(\Gamma)$.

Note that the converse statement of the last sentence is not true in general as there are weighted games which are not self-dual. 


\section{Computational complexity of related problems}

First, we present a simple game with $2 n$ players, dimension $n$ and codimension $2^{n-1}$. Other examples of simple games with $2 n$ players and dimension $2^{n-1}$ can be found in $[12,11]$.

Example 1. Given a positive integer n, Freixas and Marcinicak (Theorem 2 of [3]) define a simple game with $2 n$ players and dimension $n$. Let $\Gamma=$ $(N, \mathcal{W})$ be a simple game defined by $N=\{1,2, \ldots, 2 n\}$ and $S \in \mathcal{W}$ iff $S \cap\{2 i-1,2 i\} \neq \emptyset, i \in\{1, \ldots, n\}$, then $\Gamma$ has dimension $n$,

$\Gamma=[1 ; 1,1,0,0, \ldots, 0,0] \cap[1 ; 0,0,1,1,0,0, \ldots, 0,0] \cap \ldots \cap[1 ; 0,0, \ldots, 0,0,1,1]$

As $S$ is a winning coalition in $\Gamma^{*}$ iff $N \backslash S$ is a losing coalition in $\Gamma, \Gamma^{*}=$ $\left(N, \mathcal{W}_{1}^{*} \cup \ldots \cup \mathcal{W}_{n}^{*}\right)$, where $\mathcal{W}_{i}^{*}=\{S \subseteq N:\{2 i-1,2 i\} \subseteq S\}, i \in\{1, \ldots, n\}$. As $\Gamma^{*}$ is a composition of $n$ unanimity games, $\Gamma^{*}$ has dimension $2^{n-1}$ [5] and $\Gamma$ has codimension $2^{n-1}$ (by Lemma 2).

Proposition 1. Given a simple game $\Gamma$ as union (intersection) of weighted games, computing a representation of $\Gamma$ as intersection (union) of weighted games requires exponential time.

The complexity of several problems about representations of simple games as intersections of WMGs were analyzed in [1]. We provide here a new reduction from the NP-hard Subset Sum Problem (SSP). Our reduction differs in the fact that for the associated game we know both the dimension and the codimension. Recall that in the SSP on input a finite set $A=\left\{a_{1}, \ldots, a_{n}\right\}$ of positive integers and a positive integer $b$ we want to know whether there is a subset $A^{\prime} \subseteq A$ such that $\sum_{a_{i} \in A^{\prime}} a_{i}=b$.

Let $I=\left(b ; a_{1}, a_{2}, \ldots, a_{n}\right)$ be an instance of the SSP, $d>1$, and let $\Gamma(I, d)$ be the game defined on $n+d$ players by the intersection of the $d$ WMGs:

$$
\begin{aligned}
& {\left[3 b+1 ; 3 a_{1}, \ldots, 3 a_{n}, 1,1,0,{ }^{2(d-1)}, 0\right],\left[3 b+1 ; 3 a_{1}, \ldots, 3 a_{n}, 0,0,1,1,0,{ }^{2(d-2)}, 0\right],} \\
& \ldots,\left[3 b+1 ; 3 a_{1}, \ldots, 3 a_{n}, 0,{ }^{2(d-1)}, 0,1,1\right] .
\end{aligned}
$$

Lemma 3. Let $d>1$. When $I$ is a yes instance of $\operatorname{SSP}$ then $\operatorname{dim}(\Gamma(I, d))=$ $d$ and $\operatorname{codim}(\Gamma(I, d))=2^{d}$, otherwise, $\operatorname{dim}(\Gamma(I, d))=\operatorname{codim}(\Gamma(I, d))=1$.

Proof. Let $X \subset\{1, \ldots, n\}, E=\{n+1, \ldots, n+2 d\}$ and $Y \subset E$. Let $\Gamma$ be the game given in Example 1 taking $E$ as the set of players. Observe that if $\sum_{i \in X} a_{i}>b, \sum_{i \in X} a_{i} \geq b+1$, thus $X \cup Y$ is a winning coalition in $\Gamma(I, d)$. When $\sum_{i \in X} a_{i}<b, \sum_{i \in X} a_{i} \leq b-1$, thus $X \cup Y$ is a losing coalition in $\Gamma(I, d)$. In the case that $\sum_{i \in X} a_{i}=b, X \cup Y$ is a winning coalition in $\Gamma(I, d)$ iff $Y$ is a winning coalition in $\Gamma$.

When $I$ is a yes instance of SSP it follows that $\operatorname{dim}(\Gamma(I, d))=d$ as otherwise $\Gamma$ will have a smaller dimension. On the other hand, it is easy to 
see that $\Gamma(I, d)^{*}$ is the composition of $d+1$ unanimity games and therefore $\operatorname{dim}\left(\Gamma(I, d)^{*}\right)=2^{d}$ according to [5], thus $\operatorname{codim}(\Gamma(I, d))=2^{d}$. When $I$ is a no instance, there is no $X \subseteq\{1, \ldots, n\}$ for which $\sum_{i \in X} a_{i}=b$. Therefore $X \cup Y$ is winning in $\Gamma(I, d)$ iff $\sum_{i \in X} a_{i} \geq b+1$. Thus $\Gamma(I, d)$ is the game $[b+$ $\left.1 ; a_{1}, \ldots, a_{n}, 0,2 d ., 0\right]$ and we conclude that $\operatorname{dim}(\Gamma(I, d))=\operatorname{codim}(\Gamma(I, d))=$ 1 .

Combining lemmas 3 and 2 we can prove the following results.

Proposition 2. Let $d_{1}$ and $d_{2}$ be two integers with $1 \leq d_{2}<d_{1}$. Then the problem of deciding whether the union of $d_{1}$ given WMGs can also be represented as the union of $d_{2}$ WMGs is NP-hard.

Proposition 3. Let $d_{1}$ and $d_{2}$ be two integers with $1 \leq d_{1}, d_{2}$. Then the problem of deciding whether the intersection (union) of $d_{1}$ given WMGs can also be represented as the union (intersection) of $d_{2}$ WMGs is NP-hard.

As a consequence of the previous results, given a simple game $\Gamma$ as union or intersection of WMGs, to compute $\operatorname{dim}(\Gamma), \operatorname{codim}(\Gamma)$ or deciding whether $\Gamma$ is weighted are NP-hard problems.

Recall that two game representations are said to be equivalent whenever the represented games have the same set of winning coalitions. We can extend several results on equivalence problems from [2] to games given as unions of WMG, in particular we have.

Proposition 4. Checking whether a given union of WMGs is equivalent to a given union of WMGs is co-NP-complete, even if all weights are equal to 0 or 1 .

\section{Future work}

It remains open to exhaustively classify the dimension and codimension of all complete simple game up to $n$ players. Some bounds about dimension are given by Freixas and Puente [5] and Olsen et al. [11]. As well as to find complete simple games with small dimension (codimension), but with large codimension (dimension), and to construct analytical examples with specific dimension and codimension. It is also interesting to find real simple games with large dimension or codimension as the example given by Kurz and Napel [8]. 


\section{References}

\section{References}

[1] V.G. Delneko and G.J. Woeginger. On the dimension of simple monotonic games. European Journal of Operational Research, 170:315-318, 2006.

[2] E. Elkind, L.A. Goldberg, P.W. Goldberg, and M. Wooldridge. On the dimensionality of voting games. In AAAI Conference on Artificial Intelligence, pages 69-74, 2008.

[3] J. Freixas and D. Marciniak. On the notion of dimension and codimension of simple games. Cont. to Game Theory and Management, 3:67-81, 2010.

[4] J. Freixas, X. Molinero, M. Olsen, and M. Serna. On the complexity of problems on simple games. RAIRO - Op. Research, 45:295-314, 9 2011.

[5] J. Freixas and M. A. Puente. A note about games-composition dimension. Discrete Applied Mathematics, 113(2-3):265-273, 2001.

[6] J. Freixas and M.A. Puente. Complete games with minimum. Annals of Operations Research, 84:97-109, 1998.

[7] J. Freixas and M.A. Puente. Dimension of complete simple games with minimum. European Journal of Operational Research, 188(2):555-568, 2008 .

[8] S. Kurz and S. Napel. Dimension of the Lisbon voting rules in the EU Council: a challenge and new world record. Optimization Letters, to appear, 2015.

[9] X. Molinero, M. Olsen, and M. Serna. On the complexity of exchanging. Information Processing Letters, to appear, 2016.

[10] X. Molinero, F. Riquelme, and M. J. Serna. Forms of representations for simple games: sizes, conversions and equivalences. Mathematical Social Sciences, 76:87-102, 2015.

[11] M. Olsen, S. Kurz, and X. Molinero. On the construction of high dimensional simple games. CoRR, abs/1602.01581, 2016.

[12] A.D. Taylor and W.S. Zwicker. Simple games: desirability relations, trading, and pseudoweightings. Princeton University Press, New Jersey, USA, 1999. 